This is a copy of the last reviewed version before proofs.

The final version of this article was published in

Optical Materials 33(7) 1128-1130 (2011)

DOI: 10.1016/j.optmat.2010.08.002 


\title{
Resonance modes in rare earth doped microcrystals
}

\author{
Katleen Korthout*, Philippe F. Smet, Dirk Poelman \\ LumiLab, Dept. Solid State Sciences, Ghent University, Krijgslaan 281 - S1, 9000 Gent, Belgium
}

\begin{abstract}
Alkaline earth sulfide phosphors doped with $\mathrm{Eu}^{2+}$ or $\mathrm{Ce}^{3+}$ are good candidates for wavelength conversion in LEDs for general lighting applications. A very broad emission spectrum can be obtained by preparing SrS:Eu|SrS:Ce core|shell particles. These microcrystals are rectangular cuboids and can also be efficiently excited with electrons in a scanning electron microscope. The broad emission spectrum, ranging from $450 \mathrm{~nm}$ to $650 \mathrm{~nm}$, consists of the combined emission of $\mathrm{Ce}^{3+}$ and $\mathrm{Eu}^{2+}$ centers. Using cathodoluminescence (CL) in a scanning electron microscope, evaluation of the emission characteristics of individual cuboids was possible. Sharp peaks in the CL-spectra of individual particles could be observed and described as being resonance modes. The spectral positions and the line widths of resonance modes in these core|shell microcrystals are predicted by an extension of the plane wave model, taking into account the dispersion of the refractive index and the existence of open orbits.
\end{abstract}

Keywords: resonance modes, open orbits, rectangular cavities

\section{Introduction}

Rare earth doped alkaline earth sulfides are interesting phosphors for wavelength conversion in LEDs for lighting applications [1]. In [2] micron-sized SrS:Eu ${ }^{2+}$ octahedrons were investigated with cathodoluminescence (CL) in a scanning electron microscope (SEM). Optical whispering gallery modes (WGMs) were observed in the CL emission spectra of individual particles. Strongly luminescent particles with a broad emission band are ideal for the observation of several resonance modes when the size is in the micron range. In order to increase the width of the spectral emission, one might consider doping with both $\mathrm{Eu}$ and Ce. However codoping of SrS:Eu or CaS:Eu with Ce leads to an energy transfer from the blue-green emitting $\mathrm{Ce}^{3+}$ to the orange-red emitting $\mathrm{Eu}^{2+}$. Consequently, codoped samples do not show any $\mathrm{Ce}^{3+}$ emission, instead enhanced luminescence of the $\mathrm{Eu}^{2+}$ is obtained. A solution to this problem is the physical separation of both types of dopants in a core|shell structure.

As mentioned before, we investigated these particles with CL in a scanning electron microscope. This technique enables us to evaluate the emission characteristics of individual particles. The CL-spectra of these particles exhibit sharp peaks. By modelling the particles as rectangular microresonators, we can explain these features as resonance modes in the rectangular crossections of the cuboids. These resonant modes are often called whispering gallery modes named after the Whispering Gallery in St. Paul's Cathedral and were first analysed by Lord Rayleigh [3].

Whispering gallery modes are interesting because of their applications in microcavity lasers. They originate from total internal reflections (TIR) of light at the boundaries of the cavity and

\footnotetext{
${ }^{*}$ Corresponding author

Email address: Katleen.Korthout@UGent. be (Katleen Korthout)
}

constructive interference after circulation through a typically closed orbit inside the cavity. Circular and ring-shaped optical cavities are successfully used for microlasers and add-drop filters [4-8]. The main disadvantage of ring-shaped microcavities is the short interaction length of the waves between the circular boundary and the straight waveguide devices.

Polygonal microcavities are interesting for their potential use in the same area. WGMs have been demonstrated in hexagonally shaped microcavities [5, 7, 11], octagonal crystals [10], dodecagonal crystals [9] and cubic ones [2,12]. Square and rectangular microcavities have attracted much interest because they have a large interaction length for waveguide coupling. To calculate the resonant frequencies of a cavity, Maxwell's equations have to be solved. Since the refractive index of the considered material is wavelength dependent, this has to be done at every frequency of interest. We use the Plane Wave Model (PWM) to analyse and to predict the spectral positions and the line widths of the resonance modes [5].

\section{Experimental}

The SrS:Eu|SrS:Ce core|shell particles were prepared solvothermally [13] with ethylenediamine $\left(\mathrm{C}_{2} \mathrm{H}_{8} \mathrm{~N}_{2}\right)(99 \%$ Alfa Aesar) as a solvent. This method yields micron-sized single crystalline cuboids, mainly cubes. In this work we study the emission characteristics of individual cuboids by evaluating their CL emission in a SEM [14]. A detailed analysis of the preparation and the properties of these particles will be published elsewhere. In Figure 1 the CL spectrum of an individual cuboid is given. Superposed on the broad emission spectrum sharp peaks are observed. 


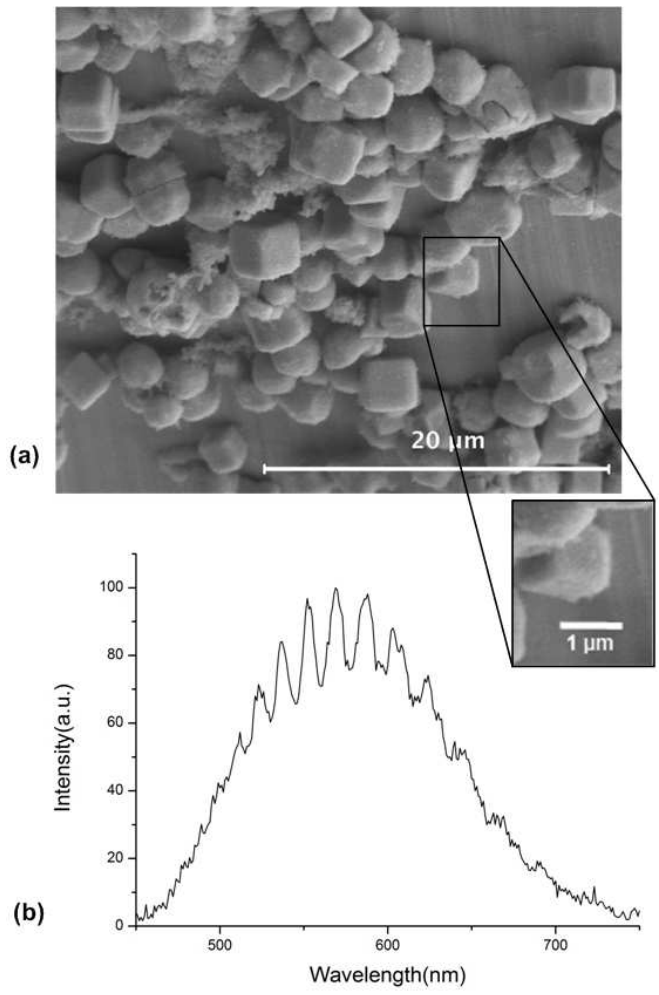

Figure 1: (a) SEM image of an ensemble of $\mathrm{SrS}: \mathrm{Eu} \mid \mathrm{SrS}: \mathrm{Ce}$ core|shell particles.(b) A CL spectrum from an individual $\mathrm{SrS}: \mathrm{Eu} \mid \mathrm{SrS}: \mathrm{Ce}$ rectangular cuboid (see inset) shows a series of peaks corresponding to the resonance modes.

\section{Theory}

Wiersig deduced the Plane Wave Model to describe resonance modes in polygonal microresonators from theoretical investigations [5]. The idea is that a light wave interferes with itself after one complete circulation in a cavity. For the trajectory of the light to be confined by TIR, the angle of incidence $\theta_{i}$ has to satisfy: $\mathrm{n} \sin \theta_{i}>1$, with $\mathrm{n}$ the refractive index of the cavity. Alternatively, $\theta_{i}$ needs to satisfy: $\theta_{c}<\theta_{i}<90^{\circ}-\theta_{c}$, where $\theta_{c}=\sin ^{-1}(1 / \mathrm{n})$ is the critical angle for TIR. The resonance modes in a 4-sided two dimensional cavity are being calculated by use of:

$$
\exp [\operatorname{inRe}(k) l] r^{2}\left(\theta_{i}\right) r^{2}\left(90-\theta_{i}\right)=1,
$$

with $l$ the total path length, $k$ the wavenumber and $r$ the appropriate Fresnel coefficient [15] (transverse magnetic (TM) or transverse electric (TE)) depending on the angle of incidence $\theta_{i}$. The formula above expresses the fact that positive interference is obtained when the phase shift is an integer multiple of $2 \pi$ after 4 bounces.

Two different types of trajectories can exist in such a resonator. Light paths that close themselves after one trajectory (left panel of Figure 2), and the ones that do not close upon themselves (right panel of Figure 2). Light trajectories with $\tan \theta_{i}=b / a$ are all closed orbits. The path lengths of different orbits defined by the same $\theta_{i}$ are identical. The path length of these closed paths is longer than those with $\tan \theta_{i} \neq b / a$. The round trip for an open orbit is defined as the meeting of wave fronts, as
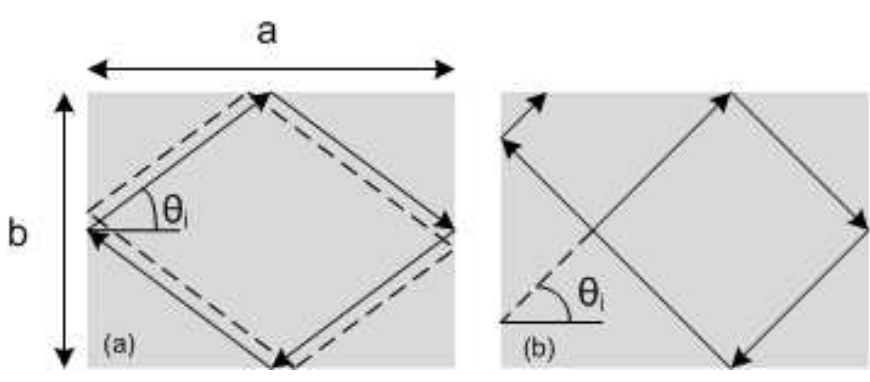

Figure 2: (a) Closed light paths in rectangular cavities with angle of incidence $\theta_{i}$, where $\tan \theta_{i}=b / a$; all closed orbits have the same path length.(b) Light paths with incident angles $\theta_{i}$ and $90^{\circ}-\theta_{i}(\tan \theta \neq b / a)$.

indicated in Figure 3. Two types of open orbits can be distinguished: (1) Almost Closed Orbits: these orbits close eventually, the angle of incidence fulfills the condition $\tan \theta_{i}=q(b / a)$ (with $q \in \mathbb{Q}$ ); (2) Real Open Orbits: these orbits never close. The open orbits give rise to a multimode spectrum [12]. To calculate the resonance modes corresponding to these orbits in the PWM, we need to consider two reflections at an angle of $\theta_{i}$ and 2 reflections at $90^{\circ}-\theta_{i}$. If one defines:

$$
\begin{gathered}
\alpha_{T M}=\frac{\sqrt{n^{2} \sin ^{2} \theta_{i}-1}}{n \cos \theta_{i}} \\
\alpha_{T M, 90}=\frac{\sqrt{n^{2} \cos ^{2} \theta_{i}-1}}{n \sin \theta_{i}} \\
\alpha_{T E}=\frac{\sqrt{n^{2} \sin ^{2} \theta_{i}-1}}{(1 / n) \cos \theta_{i}} \\
\alpha_{T E, 90}=\frac{\sqrt{n^{2} \cos ^{2} \theta_{i}-1}}{(1 / n) \sin \theta_{i}}
\end{gathered}
$$

One obtains after some basic algebraic manipulations:

$$
\begin{aligned}
& \lambda_{T M}=\frac{n l}{N+\frac{2}{\pi}\left[\arctan \left(\alpha_{T M}\right)+\arctan \left(\alpha_{T M, 90}\right)\right]} \\
& \lambda_{T E}=\frac{n l}{N+\frac{2}{\pi}\left[\arctan \left(\alpha_{T E}\right)+\arctan \left(\alpha_{T E, 90}\right)\right]}
\end{aligned}
$$

To calculate the resonant wavelengths, the path length of the trajectories of the light should be known, this is the distance between 2 consecutive wavefronts. In order to do this, the distance the light has travelled after 4 bounces is calculated and a wave front correction is added (see Figure 3). This wave front correction allows us to calculate the path length.

In the calculations only orbits whose starting position is close ( $\approx 5 \mathrm{~nm}$ ) to the position of the path after maximum 1000 bounces are taken into account, otherwise the trajectories tend to meet the corners of the cavity. These corners are typically rounded [13] or the squares are corner-cut $[2,12]$ in any practical situation; in these cases the angle of incidence at the corners does not fulfill the condition $n \sin \theta>1$ and the light will no longer be confined by TIR.

After the calculations of the resonant wavelengths, these values are binned per $1 \mathrm{~nm}$ in a histogram. The values in the histogram 

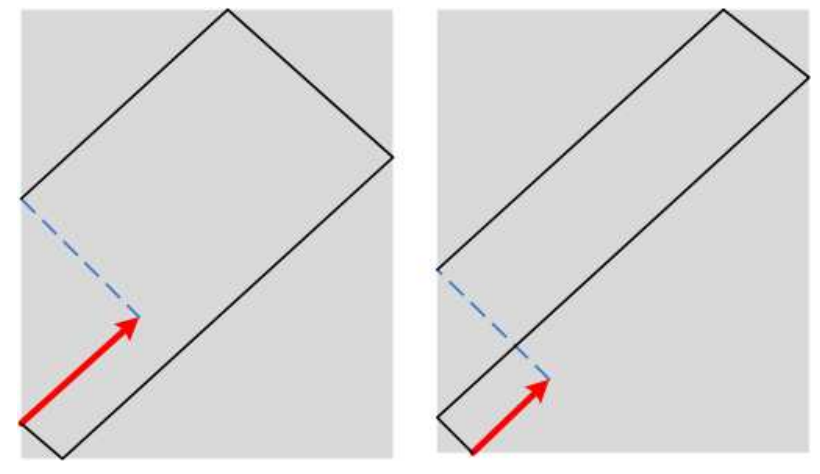

Figure 3: To calculate the optical path length one must add a wave front correction (red arrow). This correction is necessary since the path length is the distance between two consecutive wave fronts.

are homogeneously broadened using a Lorentzian distribution, to mimick the experimental resolution of a measurement. In this way the experimental spectrum can be predicted.

\section{Results and Discussions}

The CL emission spectra of individual particles show relatively sharp emission peaks (see Figure 1 (b)) superposed on the Gaussian-shaped emission of a large ensemble of core|shells. By modelling these particles as rectangular cuboids, resonance modes can occur due to light travelling in the crossections of the cuboid.

In Figure 4 the result of the simulation on a SrS:Eu|SrS:Ce rectangular cuboid is shown. We started by using equation (1)to identify the peaks in the CL spectrum, considering the refractive index of undoped $\mathrm{SrS}$ of $\approx 2.05$ at $610 \mathrm{~nm}$ [16]. To improve the fit to the experimental data, we took the wavelength dependency of the refractive index into account by using Cauchy's dispersion functions [17]. We only used the equation for TM modes, since it has been shown in [9] that WGMs are preferentially TM polarized. The simulation was performed for a rectangular cuboid with dimensions $1426 \mathrm{~nm} \times 1597 \mathrm{~nm} \times 1597 \mathrm{~nm}$, the resonance modes occured as well in the squared as in rectangular crossections. The obtained dimensions of the cuboid agree well with the values obtained from the SEM picture (see the inset in Figure 1 (b)).

If the open orbits are not taken into account, the resonance modes are not broad enough (see the dashed line in Figure 4). By considering the open orbits we are able to explain the line width and the line shape of all resonance modes in the spectrum. In [18] the authors attribute the asymmetric line shape to the interference between a resonance and the coherent background from the used laser source. Since we use an electron beam as source, there is no coherent background in our experiments.

It should be noted that we do not get an absolute intensity for the resonant wavelengths but a relative one out of the histogram. This relative intensity is multiplied by a factor depending on the expected intensity at that specific wavelength of the global CL spectrum to mimick the absolute intensity.

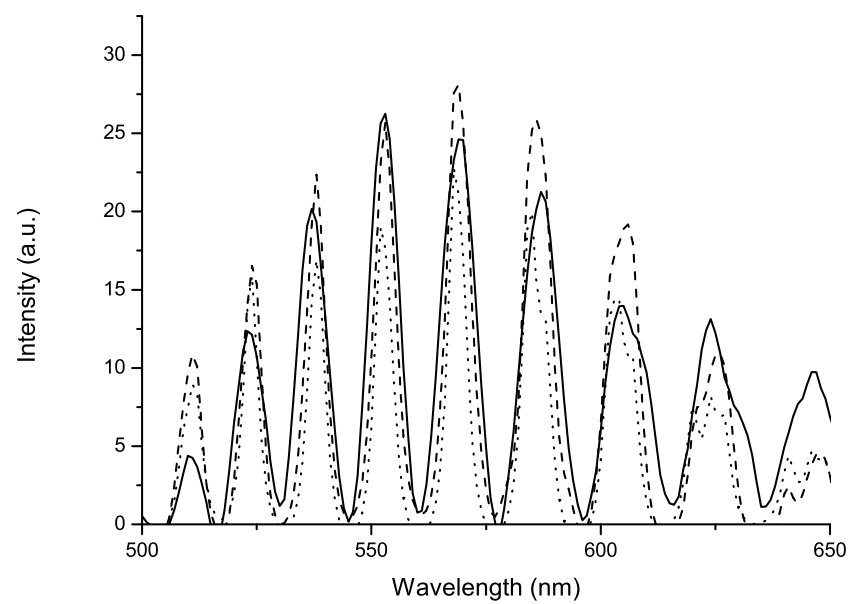

Figure 4: Difference plot between the CL spectrum of an individual $\mathrm{SrS}: \mathrm{Eu} \mid \mathrm{SrS}: \mathrm{Ce}$ rectangular cuboid and the global CL spectrum of a large ensemble (solid line). Results of the simulation taking only the closed orbits into account and the spectral resolution of the spectrometer (dashed line). The simulation of the spectrum whereby also the occurrence of open orbits is taken into account has broader peaks (dotted line).

\section{Conclusions}

We extend the plane wave model for WGMs by including open orbits in the calculations. As such we are able to explain the line width and line shape of the optical resonance modes of individual $\mathrm{SrS}: \mathrm{Eu} \mid \mathrm{SrS}: \mathrm{Ce}$ core shell particles.

\section{Acknowledgements}

Katleen Korthout is a research assistant and Philippe F. Smet is a postdoctoral research fellow of the Fund for Scientific Research-Flanders (FWO-Vlaanderen).

\section{References}

[1] P. F. Smet, I. Moreels, Z. Hens, D. Poelman, Materials 3 (2010) 2834.

[2] K. Korthout, P. F. Smet, D. Poelman, Appl. Phys. Lett. 94 (2009) 051104.

[3] J. Strutt, The Theory of Sound, New York, Dover, 1945.

[4] S. L. McCall, A. F. Levi, R. E. Slusher, S. J. Pearton, R. A. Logan, Appl. Phys. Lett. 60 (1992) 289.

[5] J. Wiersig, Phys. Rev. A 67 (2003) 023807.

[6] U. Vietze, O. Krauss, F. Laeri, Phys.Rev.Lett. 81 (1998) 4628.

[7] I. Braun, G. Ihlein, F. Laeri, J. U. Nockel, G. Schulz-Ekloff, F. Schuth, U. Vietze, O. Weiss, D. Worhrle, Appl.Phys. B 70 (2000) 335.

[8] K. J. Vahala, Nature 424 (2003) 839.

[9] T. Nobis, M. Grundmann, Phys. Rev. A 72 (2005) 063806.

[10] P. Lee, T. Lu, F. Tsai, T. Lu, H. Kuo, Appl. Phys. Lett. 88 (2006) 201104.

[11] T. Nobis, E. M. Kaidashev, A. Rahm, M. Lorenz, M. Grundmann, Phys.Rev. Lett. 93 (2004) 103903.

[12] A. W. Poon, F. Courvoisier, R. K. Chang, Opt. Lett. 26 (2001) 632.

[13] J. Van Haecke, P. F. Smet, K. De Keyser, D. Poelman, J. Electrochem.Soc. 150 (2003) G163.

[14] A. Parmentier, P. F. Smet, F. Bertram, J. Christen, D. Poelman, J.Phys. D: Appl. Phys. 43 (2010) 085401.

[15] E. Hecht, Optics, 4th Edition, Addison-Wesley, 2002.

[16] D. Poelman, D. Wauters, R. L. Van Meirhaeghe, F. Cardon, Thin Solid Films 350 (1999) 67. 
[17] J. Z. Liu, S. Lee, Y. H. Ahn, J. Y. Park, K. H. Koh, K. H. Park, Appl.Phys. Lett. 92 (2008) 263102.

[18] H. T. Lee, A. W. Poon, Opt. Lett. 29 (2004) 5. 